\title{
Backtracking depth-resolved microstructures for crystal plasticity identification - Part 2: Identification
}

\author{
Qiwei Shi ${ }^{1,2, *}$, Félix Latourte ${ }^{2}$, François Hild ${ }^{1}$, Stéphane Roux ${ }^{1}$ \\ 1: LMT, ENS Paris-Saclay / CNRS / Université Paris-Saclay, \\ 61 avenue du Président Wilson, F-94235 Cachan (FRANCE) \\ 2: EDF R\&D, Site des Renardières, \\ avenue des Renardières, Ecuelles, F-77818 Moret-sur-Loing (FRANCE) \\ Received: date / Accepted: date
}

Email: qshi@lmt.ens-cachan.fr

\begin{abstract}
The present study considers the identification of crystal plasticity parameters from the knowledge of a deformed microstructure, which has been backtracked to the reference state, and known kinematics along the sample surface. This theoretical question is tackled on a numerical (synthetic) test case. A 2D microstructure with one dimension along the depth is generated, and deformed using known crystal plasticity law. A procedure is proposed to perform the calibration of the constitutive parameters addressing the specific challenge of having a partial knowledge of the boundary conditions. The proposed identification strategy combined with the estimation of the reference microstructure is shown to retrieve the constitutive parameters with good accuracy.
\end{abstract}

Keyword: Crystal plasticity, finite element model updating, boundary conditions

\section{Introduction}

The present study aims at addressing the feasibility of backtracking the 3D depth-resolved microstructure of the final state to the initial one, in order to enhance the reliability of the interpretation of surface characterizations of mechanical tests performed in-situ (i.e., in a scanning electron microscope). The surface deformation of sample surfaces can be measured thanks to full-field measurement techniques such as Digital Image Correlation [1, 2, 3, 4]. This detailed information is specific from the observed surface, yet without a reliable 3D microstructure it remains incomplete for mechanical models. In a companion paper [5], the feasibility of the determination of undeformed microstructures from postmortem microstructures was studied. It has been shown through a $2 \mathrm{D}$ virtual test that an approximate, yet accurate, picture of the depth resolved microstructure of the initial state can be achieved. The present study focuses on the effect of this reconstructed reference on identification results.

The paper is organized as follows. Section 2 details the proposed procedure to combine the determination of the reference configuration and the calibration of material parameters. Section 3 is dedicated to a virtual experiment carried out to validate the procedure.

\section{Proposed procedure}

\subsection{Definition of the problem}

Modeling in-situ mechanical tests at the microstructure scale requires two types of unknowns, namely, the reference configuration $\Omega_{r e f}$ of the microstructure and the constitutive equation parameters $\{\boldsymbol{p}\}$ (assuming the constitutive law itself is either known or chosen). Identification consists in providing estimates of the unknown $\Omega_{r e f}$ and $\{\boldsymbol{p}\}$. An estimate of the former can be obtained when the microstructure is known in the final deformed configuration, and when $\{\boldsymbol{p}\}$ is known [5]. This paper proposes to solve the problem of determining both microstructure and constitutive parameters iteratively, assuming one set of unknowns and estimating the other up to stationarity. Those two steps are carried out alternatively until convergence of both. The whole procedure is summarized in Figure 1 and detailed in following subsections. The part 
of the flowchart in the dashed red box aims at microstructure backtracking at given constitutive parameters, as detailed in a companion paper [5]. The right part determines the constitutive parameters at fixed configuration, which is described herein.

The global loop shown in Figure 1, is indexed by a simple superscript, $m$. Each left or right block also contains iterations labeled internally with an index $(n)$ in between parentheses. At iteration $m, \Omega_{r e f}^{m}$ and $\left\{\boldsymbol{p}^{m}\right\}$ are input data. $\Omega_{r e f}^{m}$ is updated to $\Omega_{r e f}^{m+1}$ and $\left\{\boldsymbol{p}^{m}\right\}$ remains unchanged in the estimation of reference configuration [5], then $\left\{\boldsymbol{p}^{m}\right\}$ is updated to $\left\{\boldsymbol{p}^{m+1}\right\}$ and $\Omega_{r e f}^{m+1}$ remains unchanged in the calibration procedure. The global loop is stopped when a stationarity condition is met, which is based on the relative incremental correction norm between iterations $m+1$ and $m$. As mentioned in the first part of the study [5], it is assumed that the algebraic form of the law and the relevant internal parameters are correct and only parameters $\{\boldsymbol{p}\}$ are to be identified.

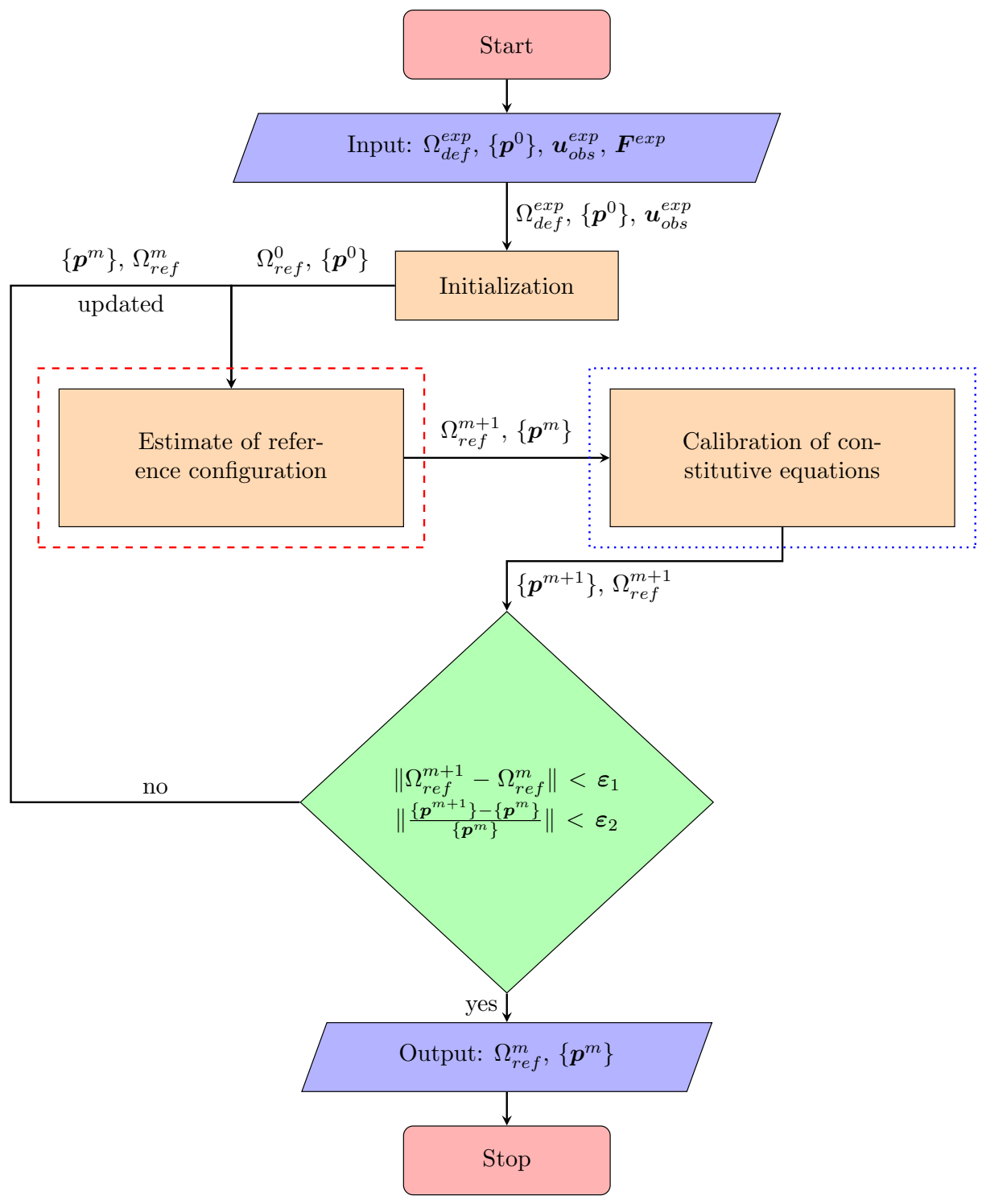

Figure 1: Flowchart summarizing the determination of the reference configuration of the microstructure and the parameters of the chosen constitutive equation

\subsection{Calibration of material parameters}

Finite Element Model Updating (i.e., FEMU [6]), which is described in Figure 2, will be used to calibrate material parameters. The FEMU algorithm is based on measured and computed displacement fields $\boldsymbol{u}$, and applied load $\boldsymbol{F}$ data. Such approaches have already been used for analyses and calibration purposes of crystal plasticity models [3, 4]. It will be referred to as FEMU-UF. In this procedure, the inputs are: 
- the reference configuration $\Omega_{r e f}^{m}$, which is assumed to be known and is not updated;

- an initial estimate of the material parameters $\left\{\boldsymbol{p}^{(n)}\right\}$ that will be updated iteratively (index $n$ );

- boundary conditions prescribed on the reference configuration during FE analyses;

- experimental kinematic field $\left\{\boldsymbol{u}^{e x p}\right\}$, experimental loading force $\left\{\boldsymbol{F}^{e x p}\right\}$.

The outputs are an updated estimate of the material parameters, which are based on the current reference configuration $\Omega_{r e f}^{m}$.

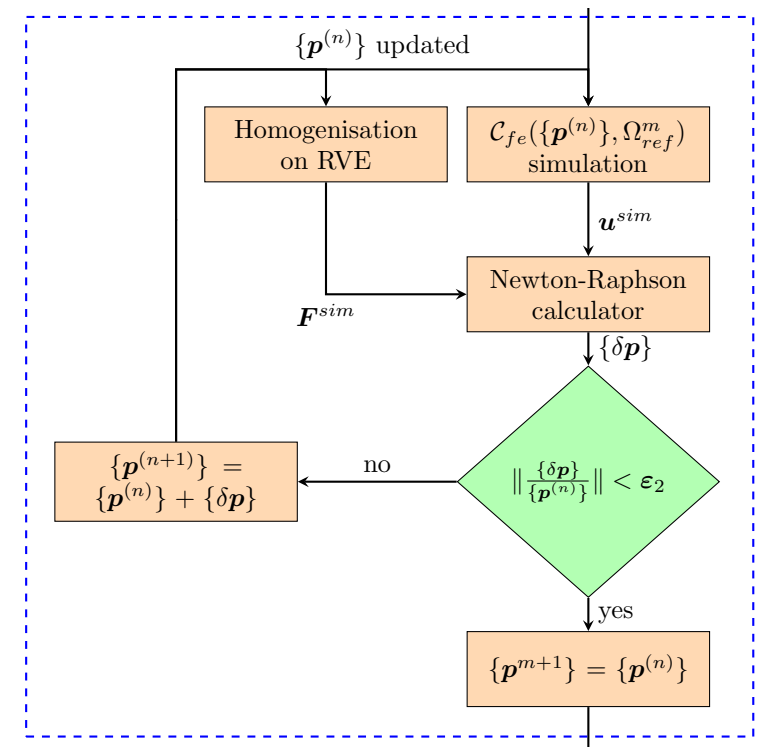

Figure 2: Flowchart describing FEMU-UF

FEMU-UF consists in minimizing a global cost function $[3,4]$

$$
\chi\left(\left\{\boldsymbol{p}^{m}\right\}\right)=\frac{1}{2 \eta_{f}^{2} N_{d o f} N_{t}} \sum_{t}\{\boldsymbol{\delta} \boldsymbol{u}\}_{t}^{T}[\boldsymbol{M}]\{\boldsymbol{\delta} \boldsymbol{u}\}_{t}+\frac{1}{\eta_{F}^{2} N_{t}}\{\boldsymbol{\delta} \boldsymbol{F}\}^{T}\{\boldsymbol{\delta} \boldsymbol{F}\}
$$

where $N_{\text {dof }}$ the number of (scalar) degrees of freedom of the surface mesh, $N_{t}$ the number of time steps, $\{\boldsymbol{\delta} \boldsymbol{u}\}_{t}$ is the column vector of the difference evaluated at surface mesh node between experimental $\left\{\boldsymbol{u}^{e x p}\right\}_{t}$ and simulated $\left\{\boldsymbol{u}^{\text {sim }}\left(\left\{\boldsymbol{p}^{m}\right\}\right)\right\}_{t}$ displacements at time step $t .[\boldsymbol{M}]$ is the DIC matrix [7], whose inverse characterizes the covariance matrix of the measured degrees of freedom [8]. When displacement and load data are taken into account (hence the acronym UF for the FEMU algorithm used herein), $\{\boldsymbol{\delta} \boldsymbol{F}\}$ is the column vector of the load differences between measured and computed values. $\eta_{f}$ and $\eta_{F}$ are the standard deviations of noise of the experimental images, and load measurement, respectively.

Boundary conditions of the FE calculation are a crucial point here again, in a similar spirit as for determining the initial microstructure [5]. For the hidden boundaries that are not accessible a prescription is used to extrapolate the displacement from those measured at the boundary of the observed surface (the discussion about those prescriptions is deferred to Section 3). The observed surface is left traction-free (i.e., Neumann condition). This is sufficient for the computation and because the displacements are not prescribed, the differences between computed and observed values, $\{\boldsymbol{\delta} \boldsymbol{u}\}_{t}$ can be used to drive the identification using FEMU-UF.

\section{Proof of concept}

\subsection{Virtual experiment}

In the following the 2D synthetic test case detailed in the first part of the study [5] is considered to prove the feasibility of the proposed procedure by comparing the results $\Omega_{r e f}$ and $\{\boldsymbol{p}\}$ with known values. A constitutive law based on dislocation dynamics for body centered cubic crystals (DD_CC [9]) has been chosen in the FE simulations. The parameters adopted in DD_CC correspond to a pressure vessel reactor steel and were obtained by identification on a stress-strain curve of ASTM A508 cl3 (16MND5) steel at room temperature [10]. For the sake of representativity, two virtual experiments have been computed, corresponding to the same 2D microstructure and boundary conditions, with two different samplings of 
orientations per grain. They are referred to as "test case 1" and "test case 2". These finite element computations are performed under plane strain assumption. The entire domain is subjected to a monotonic uniaxial tensile strain up to $6 \%$ with the following boundary conditions. The left and right edges both have uniform displacements in the $x$ (horizontal) direction.

All data extracted from the entire-domain simulations are designated as "experimental" in the following, for example the "experimental" surface kinematic field $\boldsymbol{u}^{\text {exp }}$. The "experimental" macroscopic load $\boldsymbol{F}^{\text {exp }}$ is estimated independently by integration on the edge subjected to tension from a numerical homogenization on a Representative Volume Element (RVE) $[3,11,4]$, as shown in Figure 3, with the reference parameters of DD_CC. The standard deviation of image noise, $\eta_{f}$, is set to $2 \%$ of the gray level range of the "experimental" image of the surface and $\eta_{F}$, the standard deviation of macroscopic load measurement is set to $2 \mathrm{~N}$.

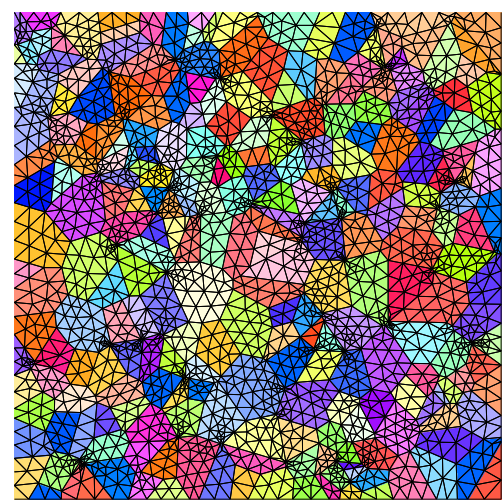

Figure 3: 2D RVE model for the calculation of macroscopic load. It consists of 250 Voronoi-style grains

The same RVE is used to calculate simulated macroscopic loads $\left\{\boldsymbol{F}^{\text {sim }}\right\}$ during the identification procedure with the current estimate of material parameters $\left\{\boldsymbol{p}^{m}\right\}$. The simulated kinematic field $\boldsymbol{u}^{\text {sim }}$ is obtained by FE calculation on the reference configuration $\Omega_{r e f}$, and the adopted boundary conditions are shown in Figure 4(b) of the companion paper [5].

Two parameters, $\tau_{F}$ and $K_{\text {self }}$, are chosen as the sought constitutive parameters, namely, $\tau_{F}$ influences principally the initial yield stress and $K_{\text {self }}$ affects the hardening ratio. Unlike other parameters in DD_CC (e.g., lath size, average obstacle diameter, Burgers vector), these two parameters cannot be determined directly from physical measurements. Their values have to be identified, and they are natural candidates for assessing the feasibility of the present procedure. Reference values are set to $\tau_{F}=35 \mathrm{MPa}$ and $K_{\text {self }}=100$ (dimensionless). Convergence is obtained when the relative variations of each parameter are less than $0.5 \%$ between two successive iterations.

To provide fair comparisons with the proposed procedure, $\Omega_{r e f}^{\text {est }}$ labeled "est" for estimated, two other models are adopted. First, the reference microstructure $\Omega_{r e f}^{e x p}$, the "ideal" one, which is not available in real-life experiment except on the sample observation surface, corresponds to the limit case where the determination of the microstructure would be exact, and, second, a quasi 2D extruded model $\Omega_{r e f}^{2 D}$ based on the microstructure of the observation surface that is simply considered as invariant along the depth as shown in Figure 4. The latter is very commonly used [4] for identification purposes from in-situ SEM experiments. 


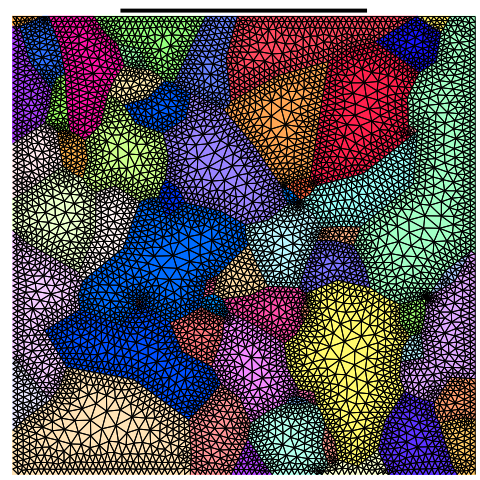

(a)

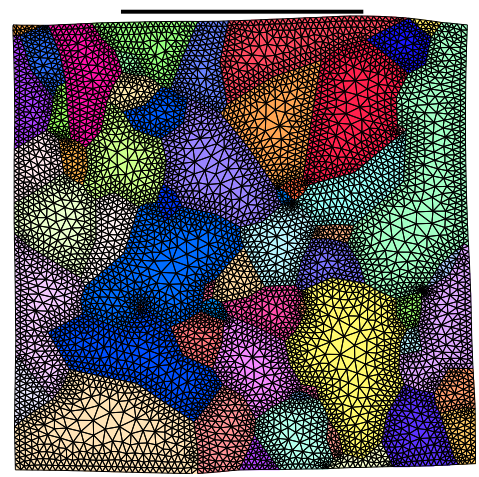

(b)

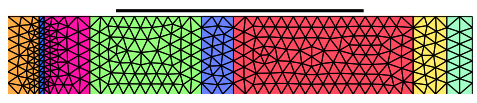

(c)

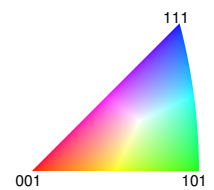

Figure 4: Microstructures used for identification purposes. (a) $\Omega_{r e f}^{\text {exp }}$; (b) $\Omega_{\text {ref }}^{\text {est }}$; (c) $\Omega_{\text {ref }}^{2 D}$. The top black lines indicate the observed ROI surface used for displacement measurement, which will be discussed in Section 3.2

\subsection{Choice of metric for $u$}

The FEMU-UF method calls for a comparison between computed and observed displacement fields on the observation surface, and an L2-norm of the difference was mentioned as a standard. However, in contrast with usual applications of FEMU [6], boundary conditions in the depth are known to be approximate at best. This implies that the displacement on the observation surface cannot be securely compared with the computed values.

To illustrate this point, Figures 5(a) and 5(c) display, respectively for the two chosen cases, displacement fields $u_{x}$ as functions of the surface coordinates $x$. They correspond to three different inferred microstructures, namely, exact $\Omega_{r e f}^{e x p}$, estimated with the current procedure $\Omega_{r e f}^{e s t}$, and extruded $\Omega_{r e f}^{2 D}$. For these computations, the DD_CC constitutive law is chosen with the exact parameters. The boundary condition prescription was chosen to be same as that of Ref. [5]. Over the observed surface, all edge displacements (i.e., in the present 2D case, the displacements at the two end points) coincide with the "experimental" data (shown in green), as a result of the prescription of boundary conditions.

However, when using the exact microstructure $\Omega_{r e f}^{e x p}$, and exact constitutive law, the computed displacement over the observed surface (shown in blue) differs significantly from the "experimental" data. This result emphasizes the large impact of the boundary conditions in the depth. For the experimental case, the displacements along the ROI boundaries results from the elastoplastic behavior of the surrounding grains. In contrast, the displacement profile computed with the exact microstructure is obtained for the boundary condition prescription, and $u_{x}$ for instance is uniform along the vertical boundaries.

It is also to be observed in the same figures that the displacement fields from the "estimated" microstructure (shown in red) give a result that is extremely close to that of the exact microstructure. In contrast, the extruded microstructure, $\Omega_{r e f}^{2 D}$, gives rise to displacement profiles that are rather distinct. However, in a blind application of this method in real life, only the experimental data are available and hence the metric to be chosen for comparing different displacement profiles or fields is critical for estimating the correct constitutive parameters.

When considering only the central zone of the observation surface, the sensitivity with respect to erroneous boundary conditions is reduced, and the local microstructure imprints its signature in a more significant way. Thus it is proposed to focus on the central half of the ROI surface $x_{1}<x<x_{2}$, with $x_{1}=L / 4$ and $x_{2}=3 L / 4$, where $L$ is the initial length of the ROI surface. When extracting only this central part, the mean translation and mean strain over the surface are different. In order to compare these profiles, a linear rescaling is proposed so that the displacements at the end of the considered comparison window coincide. More precisely, any displacement profile $v_{x}(x)$ is rescaled to $w_{x}(x)$ as

$$
w_{x}(x)=u_{x}\left(x_{1}\right)+\left(v_{x}(x)-v_{x}\left(x_{1}\right)\right) \frac{\left(u_{x}\left(x_{2}\right)-u_{x}\left(x_{1}\right)\right)}{\left(v_{x}\left(x_{2}\right)-v_{x}\left(x_{1}\right)\right)}
$$

where $u(x)$ refers to the experimental data. It is noteworthy that this rescaling is not exact, but it assumes that the local relative strain varies in proportion to its mean (just as if the localization operator were insensitive to the total amount of plastic strain), which may be considered as a decent approximation. After such rescaling over the comparison window the displacement profiles are shown in Figures 5(b) and 5(d) for test cases 1 and 2, respectively. Apart from the extruded quasi-2D microstructure that shows a poor agreement, the experimental data are very well captured by the exact and estimated microstructures for the two test cases. Therefore, it is proposed in the following to use this comparison window after rescaling as a substitute to the L2-norm used in the FEMU-UF cost function. Let us also point out that the 2D 
nature of this "toy model" restricts the comparison to a rather narrow interval, and thus few crystals, and in 1D. As a consequence, the $1 \mathrm{D}$ comparison window bears little information as compared to a more realistic 2D surface case.

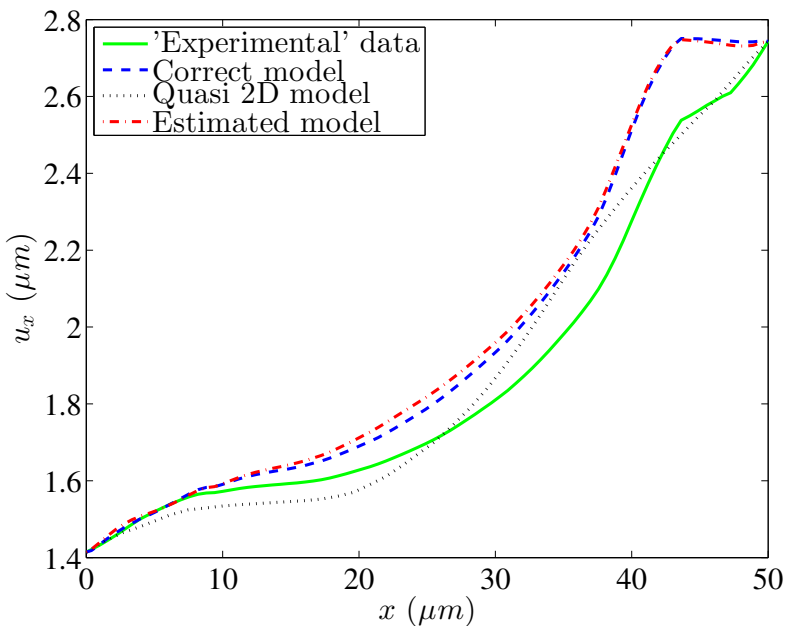

(a)

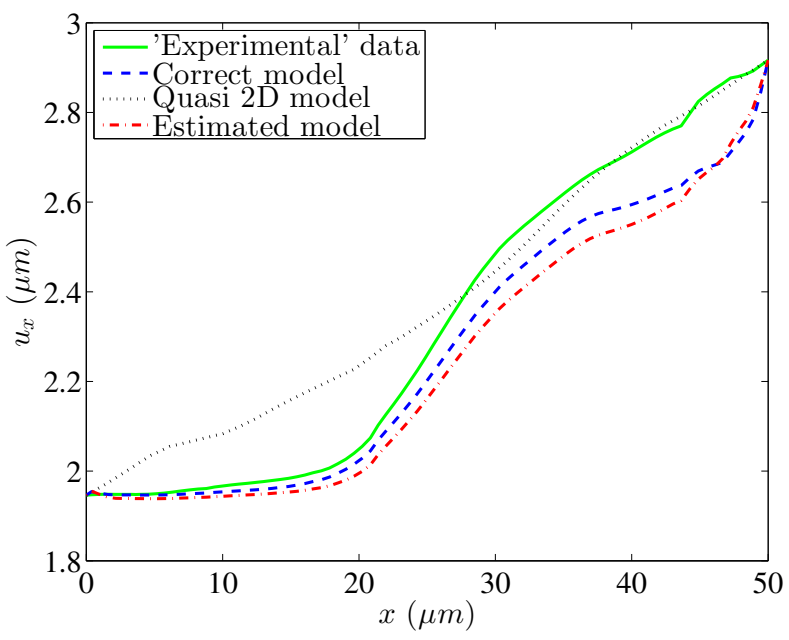

(c)

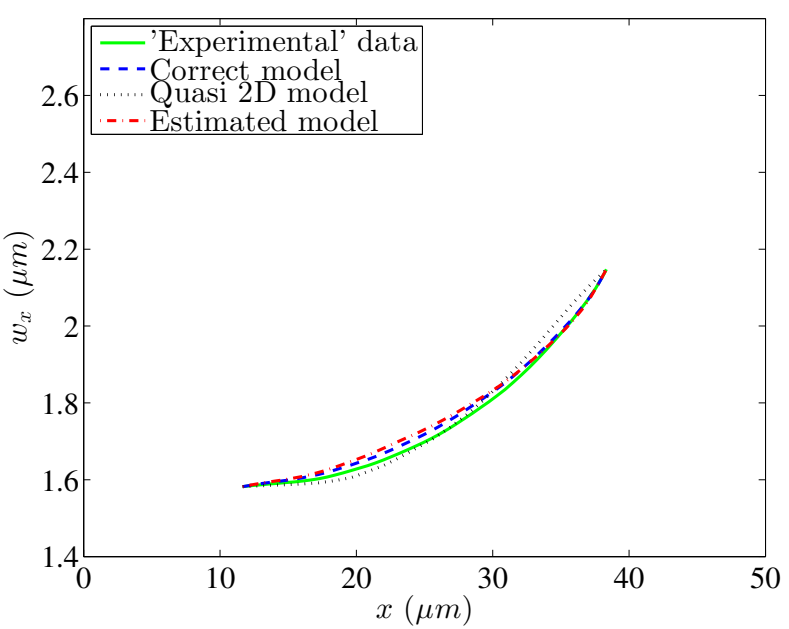

(b)

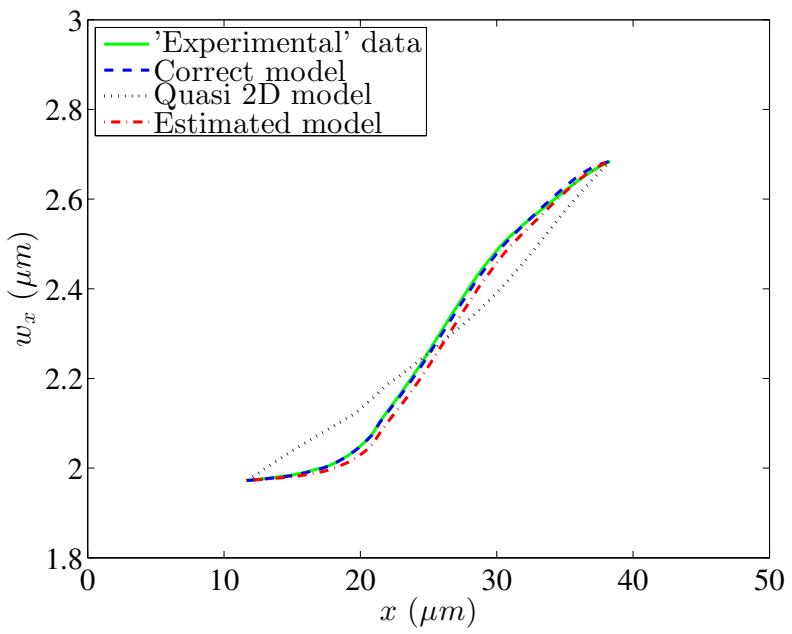

(d)

Figure 5: Surface displacement profiles as functions of coordinates $(x)$ along the observation surface for all models calculated with the exact DD_CC parameters. Top row (a-b) corresponds to test case 1, and bottom row (c-d) to test case 2. The left plots (a-c) correspond to the displacement profiles computed for all microstructures and the "experimental" data (green). The right plots (b-d) show the same profiles over a narrower comparison window and rescaled to match the experimental data at the two end points

Section 3.3 will now address the complete problem of estimating the initial configuration and identification of the constitutive parameters. To this aim, only test case 2 is selected as its microstructure appears to be more influential on the displacement profile and hence discriminating.

\subsection{Results}

The identification results are shown in Figure 6 . It can be seen that convergence is reached after $n \approx 4-5$ iterations for all the models, which is relatively fast. Three outer iterations are needed, i.e., $m=3$, for the estimated model to reach convergence. The correct and estimated models lead to very good identification results, whereas model $\Omega_{r e f}^{2 D}$ gives much poorer results. The efforts for having a more faithful picture of the in-depth microstructure are rewarded by the accuracy of the identification results for constitutive parameters. 


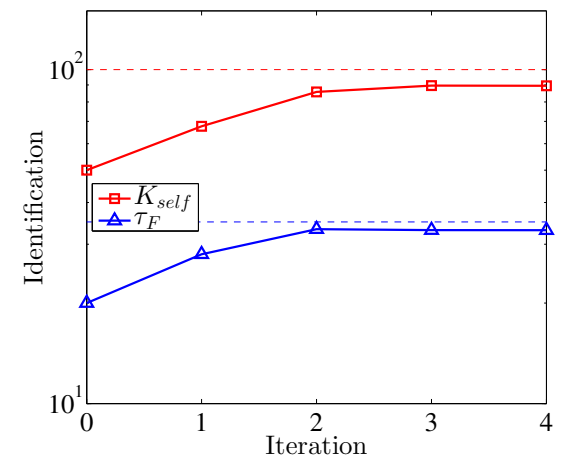

(a)

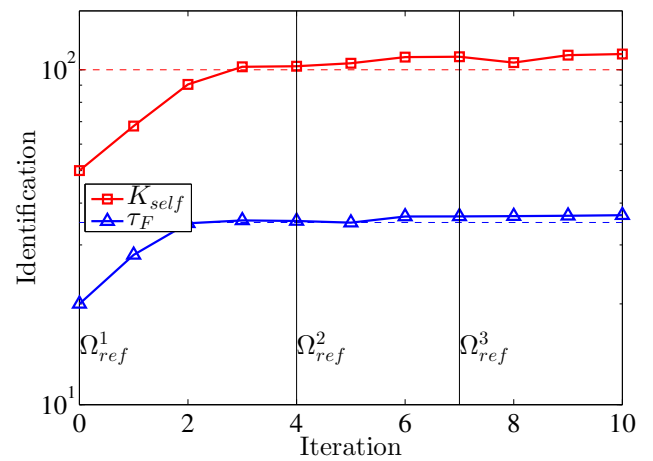

(b)

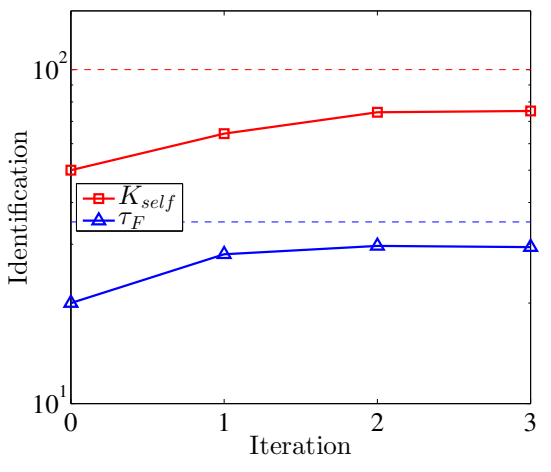

(c)

Figure 6: Calibration results for $\tau_{F}$ and $K_{\text {self }}$ for different microstructures: (a) exact; (b) estimated; (c) quasi 2D configurations. The reference values are shown as dashed lines

The changes of the cost function during the identification iterations are shown in Figure 7. As shown in Figure 7(b), since the microstructure changes from $\Omega_{r e f}^{1}$ to $\Omega_{r e f}^{2}, \Phi_{u}$ drops significantly, although using the same parameters of DD_CC law. This phenomenon illustrates the beneficial effect of updating the reference configuration. The drop is much smaller in subsequent transitions. At convergence the estimated model leads to comparable displacement residuals $\Phi_{u}$ to the correct model, both much lower than $\Omega_{\text {ref }}^{2 D}$ (i.e., quasi $2 \mathrm{D}$ model). This is another proof that having a better knowledge of the in-depth microstructure brings better synergy between experiments and simulations.

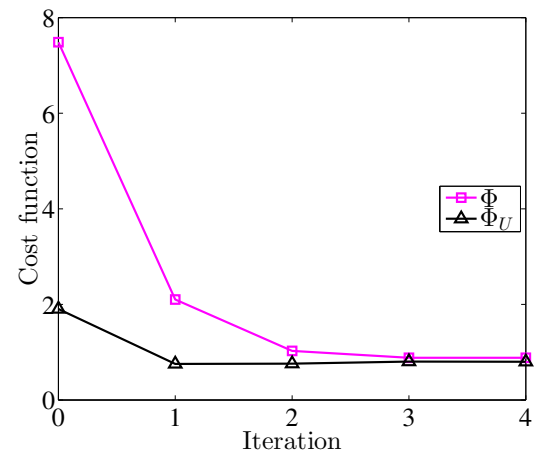

(a)

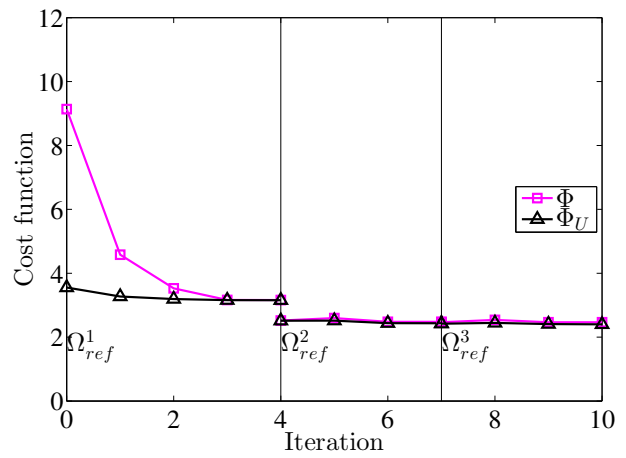

(b)

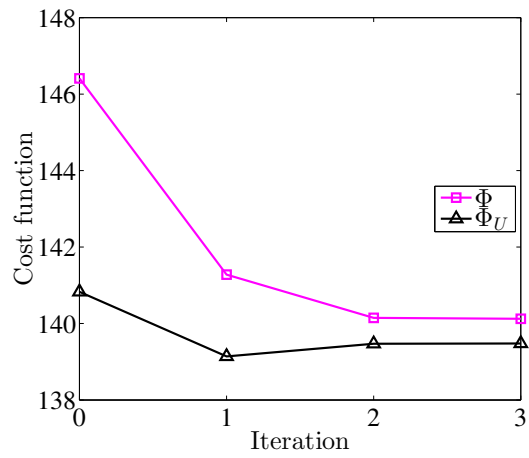

(c)

Figure 7: Cost function changes during the identification for each model: (a) exact; (b) estimated; (c) Quasi 2D configurations

In terms of deviation from the correct reference configuration $\Omega_{r e f}^{e x p}, \Omega_{r e f}^{3}$ is $23 \%$ lower than $\Omega_{r e f}^{0}$ and $57 \%$ lower than $\Omega_{d e f}^{e x p}$. In other words, the procedure that combines the determination of the reference configuration and the calibration of material parameters gives satisfactory results in this virtual experiment.

\section{Conclusion}

The present study discussed the feasibility of combining the determination of undeformed microstructures from postmortem microstructures [5] and the calibration of material parameters. An iterative process has been proposed to tackle the two problems one at a time until global convergence. A 2D model has been adopted to carry out the analysis of a virtual experiment. It has been found that even though the estimated microstructure differs from the reference one, the identification results based on it are significantly better than with the commonly used (i.e., extruded) model. From the better reconstructed model and with all information available at the surface, crystal plasticity can be studied more precisely.

More efforts should be devoted to provide better descriptions of boundary conditions in the depth of the material, as they are the current limitation to further lower the discrepancy with the exact reference configuration. An option is to treat the in-depth boundary conditions as unknowns to be calibrated [12]. Another problem to address is the heavy 
calculation cost, as the procedure proposed herein contains nested loops of FE calculations. An important way to reduce the time cost is to reduce the dimension along the depth, and special attention must be paid to avoid compromising the whole model.

It should be noted that this study is devoted to a feasibility check, and hence a synthetic two-dimensional example is considered. The efficiency and correctness of the process for three-dimensional models should be validated in simulations and, all the more importantly, with experimental data. The strategy proposed herein is adapted to both 2D and 3D cases. The obvious additional complexity of the 3D topology as compared to $2 \mathrm{D}$ situations is not expected to cause extra difficulties neither in the backtracking procedure, nor in the calibration step.

\section{Acknowledgments}

The authors acknowledge the financial support of Euratom research and training program 2014-2018 SOTERIA under grant agreement No. 661913. This paper reflects only the authors' view and that the Commission is not responsible for any use that may be made of the information it contains. They also thank Dr. Adrien Guery for constructive advices. 


\section{References}

1. A. Allais, M. Bornert, T. Bretheau, and D. Caldemaison. Acta Metall. Mater., 42(11), 3865 (1994).

2. T. Hoc, J. Crépin, L. Gélébart, and A. Zaoui. Acta Mater., 51(18), 5477 (2003).

3. E. Héripré, M. Dexet, J. Crépin, L. Gélébart, A. Roos, M. Bornert, and D. Caldemaison. Int. J. Plast., 23(9), 1512 (2007).

4. A. Guery, F. Hild, F. Latourte, and S. Roux. Mech. Mater., 100, 55 (2016).

5. Q. Shi, F. Latourte, F. Hild, and S. Roux. LMT Cachan, France, unpublished research, 2017.

6. E. Pagnacco, A.S. Caro-Bretelle, and P. Ienny, in Full-Field Measurements and Identification in Solid Mechanics ed. M. Grédiac and F. Hild (New Jersey: John Wiley \& Sons, Inc., 2012), p. 247.

7. F. Mathieu, H. Leclerc, F. Hild, and S. Roux. Exp. Mech., 55(1), 105 (2015).

8. F. Hild and S. Roux. Exp. Mech., 52, 1503 (2012).

9. G. Monnet, L. Vincent, and B. Devincre. Acta Mater., 61(16), 6178 (2013).

10. F. Latourte, T. Salez, A. Guery, N. Rupin, and M. Mahé. J. Nucl. Mater., 454(1-3), 373 (2014).

11. M. Kraska, M. Doig, D. Tikhomirov, D. Raabe, and F. Roters. Comput. Mater. Sci., 46(2), 383 (2009).

12. M. Bertin, C. Du, J. P.M. Hoefnagels, and F. Hild. Acta Mater., 116, 321 (2016). 